\title{
BMJ Open Sick leave and return to work for patients with anxiety and depression: a longitudinal study of trajectories before, during and after work-focused treatment
}

\author{
Kenneth Sandin (D) , ${ }^{1,2}$ Frederick Anyan, ${ }^{2}$ Kåre Osnes, ${ }^{1}$ \\ Ragne Gunnarsdatter Hole Gjengedal, ${ }^{1,2}$ Jonas Sigurd Risberg Leversen, ${ }^{1}$ \\ Silje Endresen Reme, ${ }^{3}$ Odin Hjemdal ${ }^{1,2}$
}

To cite: Sandin K, Anyan F, Osnes $\mathrm{K}$, et al. Sick leave and return to work for patients with anxiety and depression: a longitudinal study of trajectories before, during and after workfocused treatment. BMJ Open 2021;11:e046336. doi:10.1136/ bmjopen-2020-046336

- Prepublication history for this paper is available online. To view these files, please visit the journal online (http://dx.doi org/10.1136/bmjopen-2020046336).

Received 26 0ctober 2020 Accepted 13 September 2021

Check for updates

(c) Author(s) (or their employer(s)) 2021. Re-use permitted under CC BY-NC. No commercial re-use. See rights and permissions. Published by BMJ.

${ }^{1}$ Division of Mental Health and Substance Abuse,

Diakonhjemmet Hospital, Oslo, Norway

${ }^{2}$ Department of Psychology, Norwegian University of Science and Technology, Trondheim,

Norway

${ }^{3}$ Department of Psychology, University of Oslo, Oslo, Norway

Correspondence to

Kenneth Sandin;

kenneth.sandin@diakonsyk.no

\section{ABSTRACT}

Objectives Sick leave due to anxiety and depression is a heterogeneous process constituting a pressing public health issue. This longitudinal study aimed to identify sick leave trajectories among patients before, during and after work-focused treatment, in all 29.5 months We then aimed to determine the background and clinical characteristics of these trajectory groups.

Methods Background and clinical data were collected by patient self-report $(\mathrm{N}=619)$ in an observational study in a specialised mental healthcare clinic. Sick leave was recorded from national registry data. A latent growth mixture model identified trajectories. Multinomial logistic regression determined differences in background characteristics while a one-way analysis of variance (ANOVA) identified clinical differences.

Results We identified three trajectories: The 'Resilient' group (47.7\%) had low sick leave throughout the period. The two other groups ('Recovery', $31.8 \%$ and 'High risk', $20.5 \%$ ) had similar pretreatment trajectories: lower sick leave one year prior which increased to high sick leave at the start of treatment. After treatment, the 'Recovery' group made an almost full return to work while the 'High risk' group remained at high sick leave. The two groups with high sick leave had more women and higher age compared with the 'Resilient' group. All groups had similar clinical scores at the start of treatment, but the 'High risk' groups had residual depressive symptoms at the end of treatment. Effect sizes for anxiety and depression were moderate or large for all groups, (Cohen's $d=0.74-1.81$ ), and $87.2 \%$ of the total sample were fully working one year after treatment.

Conclusion We found three subgroups with distinctly different trajectories. Female gender and higher age were associated with high sick leave at the start of treatment, while residual depressive symptoms at the end of treatment predicted continued sick leave. The study points to the possibility of improving patient outcomes in the future by stratifying and tailoring treatment to patient characteristics.

\section{INTRODUCTION}

Sick leave due to common mental disorders (CMDs) such as anxiety and depression is a
Strengths and limitations of this study

- This is the first study that uses registry data spanning pretreatment and post-treatment to examine sick leave trajectories among patients with common mental disorders.

- Latent growth modelling over 2.5 years gave detailed knowledge of sick leave behaviour.

- The study contributes to a better understanding of sick leave over time with implications for treatment and research.

- The study was not a randomised controlled trial and lacks a control group, precise impact of the intervention is yet to be determined.

pressing public health issue. Globally, one in five people fulfil diagnostic criteria for a CMD at any given time, and the lifetime prevalence is estimated to almost one-third of the population $(29.2 \%) .{ }^{1}$ People suffering from mental health problems commonly report that their daily activities are impeded, and mental illness leads to low employment rates and reduced productivity. ${ }^{2}{ }^{3}$ Measured in 'years lived with disability', mental ill health is the largest contributor to burden of disease worldwide. ${ }^{4}$ The economic cost of mental disorders in Europe alone is estimated to be $€ 600$ billion per year, the majority of which comes from reduced employment and lost productivity. $^{5}$

Several policy initiatives have been launched to address the rising cost of CMD. Most well known is perhaps Improving Access to Psychological Therapies in the UK. The programme was launched to alleviate the cost of mental illness, including lost productivity, by scaling up access to psychological therapy. ${ }^{6}$ Similarly, the Faster Return (FR) programme was initiated in Norway in 2007. As Norway has the highest rates of sick leave globally, the 
target population for the programme were patients 'on or at risk of sick leave'. ${ }^{7}$ The data in the present study were collected in a clinic originally funded by the FR programme.

Offering effective treatment for CMD symptoms that also reduces sick leave is not straightforward. Reviews indicate that psychotherapy alone has little impact on sick leave, but that pairing it with work-focused interventions can help patients return to work (RTW). ${ }^{8}$ There is still room for improvement. Some studies struggle to find effect on work status, while others find that the effect is unevenly distributed among patient groups. ${ }^{9-11}$ This is not surprising, as sick leave is a heterogeneous process. Personal characteristics, features of the work, workplace and health issues including symptom severity all contribute to sick leave. ${ }^{12}{ }^{13}$ Whether this heterogeneity of prognostic factors contain more homogeneous subgroups of patients with similar risk profiles is currently not clear. If so, identifying these groups could be an important next step towards more effective treatment.

Developing more effective treatment is also likely to depend on a better understanding of how RTW is sustained over time. First, previous sick leave predicts future absence, regardless of health status, raising the question of whether treatment outcome may in part be determined by sick leave history. ${ }^{14}$ Second, longitudinal studies evaluating how patients fare after interventions show that they often struggle to increase and maintain work participation. ${ }^{15}{ }^{16}$ Cross-sectional measurements of sick leave (eg, at the end of treatment) may therefore be of limited value for understanding patient outcomes and needs. A more thorough understanding of sick leave behaviour may depend on including data before, during and after an intervention.

In the present study we therefore wanted to examine trajectories of sick leave before, during and after workfocused treatment. We then examined if known risk factors from the literature differed between the various trajectories. We included both non-modifiable background characteristics and modifiable clinical characteristics: gender, age, education level, somatic comorbidity, CMD symptom severity, self-rated subjective health and RTW self-efficacy (RTW-SE). All factors that have consistently been found to predict sick leave and RTW. ${ }^{13} 17$

This study is unique in being the first to combine clinical data with longitudinal registry based sick leave data covering both preintervention and postintervention periods for patients with CMD. Furthermore, registry data gave us an objective measure of sick leave with no loss to follow-up. The observational period stretched from one year prior to treatment to one year after end of treatment (24 months). Average duration of treatment was 5.5 months, making the total observation period 29.5 months, or approximately 2.5 years.

Our primary objective was: (1) to determine if subgroups of patients could be identified based on their sick leave trajectories before, during and after they received workfocused treatment in a specialised healthcare clinic.
Our secondary objectives were to (1) examine if these groups differed on non-modifiable background characteristics: age, gender, education and somatic comorbidity, and (2) examine if these groups differed on modifiable clinical characteristics: CMD symptom severity, self-rated subjective health and RTW-SE.

\section{METHODS}

\section{Study context}

Data were collected in an outpatient clinic at Diakonhjemmet Hospital in Oslo, Norway. The clinic is part of the specialised healthcare service and the observational study ran from 2013 to 2016. Cognitive behavioural therapy (CBT) and metacognitive therapy (MCT) are recommended for treating anxiety and depression. ${ }^{18} 19$ Our treatment consisted of short-term CBT or MCT based on diagnose-specific manuals, ${ }^{20}{ }^{21}$ which was paired with work-focused interventions. The work-focus consists of clinicians addressing the patients work status from the onset of therapy, mapping resources and barriers for maintaining work status, or in the case of sick leave, returning to work. A gradual RTW plan is developed in cooperation with the patient, and interventions tailored to address challenging issues, for example, role playing to help patients gain confidence communicating their needs in the workplace. The work-focused interventions are based on Dutch research, ${ }^{22}$ and have been further developed and adapted to a Norwegian context. They have been described in more detail in a previous publication. ${ }^{23}$ The treatment was provided by 20 therapists who were clinical psychologists, psychiatrists or psychiatric nurses.

The Norwegian welfare system ensures that patients receive $100 \%$ coverage of lost income through sick pay from day one and up to one year. Employers cover payments for the first 16 days, and the Norwegian Labour and Welfare Administration cover the remaining part of the year. Patients may then be eligible for long-term benefits that cover roughly $66 \%$ of their original income. ${ }^{24}$

\section{Procedure and participants}

Participants were referred for treatment by their general practitioners (GPs), who are responsible for certifying sick leave. To be eligible for inclusion, patients had to be adults of working age (18-67 years), either on or at risk of sick leave due to anxiety and/or depression. All patients in the study were either on sick leave or deemed 'at risk' of sick leave by their GPs. Patients on long-term benefits such as disability pension at baseline were excluded from the study. Patients were also excluded if they were suffering from psychosis (ie, bipolar disorder, schizophrenia or other psychotic disorders), engaged in active substance abuse, suffered from cluster A or B personality disorder or considered high suicide risk. Patients received oral and written information about the study and signed a consent form before being enrolled. In all, 619 patients were recruited. 
All patients were screened at an initial assessment session by a clinical psychologist and diagnosed according to the International Classification of Diseases- $10 .{ }^{25}$ After the screening session, patients waited an average of 42.71 days $(S D=29.4)$ before starting treatment, after which patients were given weekly sessions of work-focused treatment. Average number of sessions including screening was 10.71 ( $S D=3.24$, duration $M=121.92$ days, $S D=57.92)$. Average duration of entire intervention including screening was thus 5.5 months. Background variables and clinical scores were collected from self-report questionnaires during treatment at the clinic. Registry data on sick leave was collected from national registries.

\section{ETHICAL CONSIDERATIONS}

This observational study qualifies as health service research and was therefore pre-approved by the Norwegian Data Protection Authority. Patients were informed that they could withdraw consent without providing any explanation. The study was conducted according to the principles of the Helsinki Declaration. User representatives were involved in the planning of the current study, including design and data collection. The primary user representatives involved represented the user interest organisation Mental Health.

\section{MEASURES}

\section{Registry data}

Sick leave was collected from national registries containing comprehensive records as reported by medical doctors. The data covered each patient's sick leave spanning 29.5 months: One year prior to inclusion, the duration of treatment, and one year after the end of treatment. Sick leave for each individual is given as a start and end date of episode. We had information on degree of sick leave, for example, $50 \%$ or $100 \%$. However, for the purpose of this study, we operationalised sick leave as a dichotomous variable: Fully working with no sick leave of any kind vs all degrees of sick leave. We did this to give us a conservative measure of RTW, and to facilitate comparisons with other studies.

For the latent growth mixture model (LGMM), we set measurement points at 90-day intervals. Thus, we had four measurements prior to treatment: at 360 days, 270 days, 180 days and 90 days. The same intervals were applied after the end of treatment. The treatment period was included in the model as three measurement points: Screening session, start of treatment and end of treatment. Thus, the final model contained 11 measurement points (figure 1). Each measurement point reflects the sick leave of the patient population at the time. Each patient was given a value of either 0 ('Fully working') or 1 ('On sick leave') for each of the measurement points based on model estimates. The sick leave of 100 patients assigned in this manner, where 46 were working and 54 were on sick leave would thus be ' $54 \%$ '.

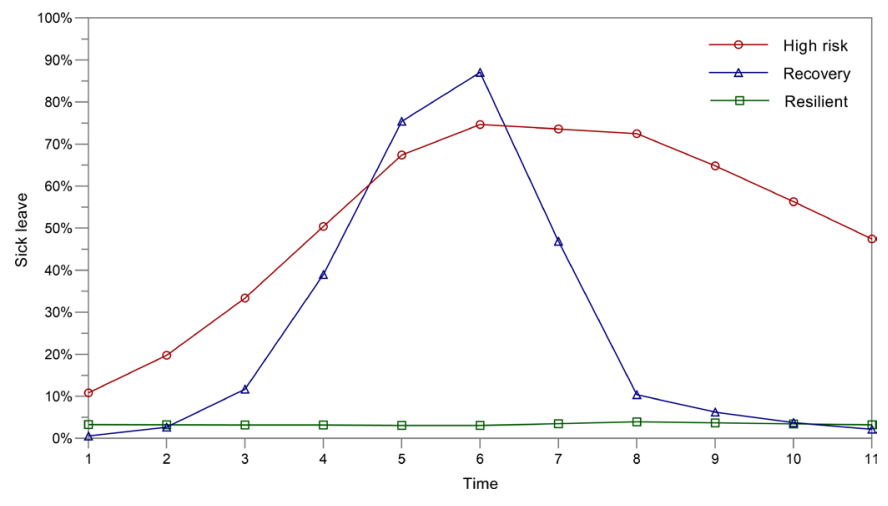

Figure 1 The three trajectories: 'High risk' 20.5\% $(n=127)$, 'Recovery' 31.8\% ( $n=197)$, 'Resilient'47.7\% ( $n=295)$. Time point 1-4 denotes the year prior to intervention at 90-day intervals. Time point 5, 6 and 7 denotes assessment, start of treatment and end of treatment. Time point 8-11 denote the year after intervention at 90-day intervals.

\section{Background characteristics}

Background characteristics were recorded as self-report at assessment and at end of treatment. Age, gender and education have all been shown to impact sick leave in previous studies. ${ }^{1326}$ In addition, comorbid somatic diagnosis have been shown to negatively impact work functioning over time in patients with CMD in a previous longitudinal study. ${ }^{17}$ The patients answered the question 'Do you have any somatic illness diagnosed by a health professional?'. Somatic diagnoses were diverse, with musculoskeletal (24.2\%), cardiovascular (12.7\%) and obesity (12.1\%), making up the main diagnostic categories. Both 'Somatic diagnosis' and 'Higher education' were included as dichotomous variables (yes/no). 'Higher education' was defined as any completed degree beyond upper secondary school, that is, the first 12 years of school.

\section{Clinical characteristics}

Anxiety and depression were measured with the Beck Depression Inventory-II (BDI-II) and the Beck Anxiety Inventory (BAI). Both questionnaires contain 21 items that are scored from 0 to 3, giving a total score of $0-63$. Higher score indicates higher symptom severity. Both the BDI-II and the BAI have demonstrated good reliability and validity. ${ }^{28}$ All patients had either a score $\geq 14$ on the BDI-II or $\geq 15$ on the BAI at baseline in keeping with criteria for clinically significant symptoms of depression or anxiety.

Self-rated health (SRH) was measured by a single item rating of total subjective health. Respondents answered the question 'How would you rate your health at the moment?' on a 4-point scale: 'Bad', 'Not so good', 'Good', 'Very good'. SRH is commonly used in public health surveys, and is a robust predictor of general health status including all-cause mortality. ${ }^{29}$

The RTW-SE questionnaire is an 11-item self-report questionnaire shown to predict RTW, including in a Norwegian sample. ${ }^{23}$ Patients are asked how they would 
deal with overcoming obstacles in RTW by responding to statements like 'I will be able to cope with potential problems at work' or 'I will be able to manage set-backs' using a 6-point Likert-scale. Total score is recorded as the mean of all responses, and higher score indicates higher self-efficacy. ${ }^{30}$

There was no missing sick leave data in the study due to the source being national registries. The same was true for the background characteristics and diagnoses, as they were recorded from patient journals. The exception was 'Higher education' with $3.1 \%$ missing values. The clinical data collected from patient self-report was also mostly complete, and more than $95 \%$ of patient questionnaires had $\leq 1$ missing item. In these cases, missing items were replaced by weighted means.

\section{Statistical analyses}

The registry data was analysed with LGMM using Mplus V.7.4. ${ }^{31}$ This method helps model unobserved heterogeneity in a population by identifying subgroups with similar individual growth trajectories across repeated measures. We favoured piecewise growth modelling, thus setting the start of treatment as the status factor reference point. Once the best fitting latent growth curve model had been established, we estimated the LGMM. To determine the appropriate class solution, we examined fit statistics with classification accuracy so that average probability of belonging to the most likely class should be high, and the average probability of belonging to the other classes should be low. ${ }^{32}$ Particular attention is given to the Bayesian Information Criterion (BIC), sample-size adjusted BIC (ABIC), and Akaike information criterion indices, Entropy values and the bootstrap likelihood ratio test (BLRT). We sought a model with lower values for the criterion indices, higher entropy values and significant $p$ values for the BLRT. ${ }^{33}$ Fit indices in combination with theoretical interpretability guided the final model selection.

Once the best fitting model had been established, we wanted to know if auxiliary variables like background and clinical characteristics differed across the groups. Only sick leave was included in the LGMM, and we analysed auxiliary variables as predictors of class membership post hoc. This is a viable approach where LGMM model entropy is $\geq 0.8 .{ }^{34}$ However, in the post hoc analyses, class membership is necessarily treated as categorical, despite assignment being probabilistic. Standard errors inherent in the parameter may thus be underestimated. It is therefore recommended to choose a more stringent significance level than $\mathrm{p}<0.05$ for these analyses. ${ }^{34}$ Significance level for all post hoc analyses in this study was thus set to $\mathrm{p}<0.01$.

Background characteristics (age, gender, education level and previously diagnosed somatic illness) were selected based on the literature and analysed as predictors using multinomial logistic regression. First, we used the 'High risk' group as the reference category. We then ran the same analyses with the 'Resilient' group as reference category, to also obtain comparisons between the 'Recovery' and 'Resilient' groups. The clinical measures are reported as pre to post sum scores. Effect size (ES) was calculated using Cohen's $d$. Between-group differences on clinical measures pre and post were analysed using a one-way analysis of variance(ANOVA) and Tukey's honestly significant difference test. ${ }^{35}$

\section{Patient and public involvement}

User representatives and patients were involved in the planning of the study, including giving feedback on data collection, the content of work-focused interventions and self-report questionnaires prior to the start of the study. Findings from the study will be disseminated in cooperation with Stiftelsen Dam, a not-for-profit trust, and Mental Health, a user interest organisation.

\section{RESULTS}

\section{Characteristics of patients}

For the total sample, average age at inclusion was 37.9 years $(S D=10.5)$. There were more women $(68.5 \%)$. More than three quarters $(76.5 \%)$ had some form of higher education. The most prevalent primary diagnoses were depressive disorders $(47.8 \%, \mathrm{n}=296)$, anxiety disorders $(36.7 \%, \mathrm{n}=227)$ and mixed anxiety and depression $(12.9 \%, \mathrm{n}=80)$. The remaining $2.6 \%$ $(n=16)$ had other primary diagnoses, such as eating or sleeping disorders (table 1).

\section{Trajectories of sick leave}

The LGMM identified a three-piece unconditional linear growth trajectory that showed adequate fit according to the root mean square error of approximation (RMSEA), the comparative fit index (CFI), and the Tucker-Lewis index (TLI): $\left(\chi^{2}=170.869, d f=43\right.$, $\mathrm{p}<0.001 ;$ RMSEA $=0.069$, (90\% $\mathrm{CI}=0.059$ to 0.080$)$; $\mathrm{CFI}=0.973$; TLI=0.966) (figure 1). Model fit indices of all unconditional LGMM models under comparison are shown in table 2.

Determining the optimal class solution was carried out incrementally until model fit ceased to improve in a 5-class solution with non-significant results for the likelihood ratio tests. Despite a lower BIC in the 4-class model than the 3-class model, classes 1 and 3 in the 4-class model were not distinct from each other as both classes followed similar trajectory. Thus, guided by theoretical interpretability, the class profile plot based on the estimated posterior probabilities and the best performing BIC, we favoured a 3-class solution whose BIC was lower than a 2-class solution. ${ }^{33}$ The 3 -class solution provided a narrower class assignment probability for most likely latent class membership than a 4-class solution, showing a higher degree of precision and reliability of the classification. Compared with the 4-class solution, the 3-class solution also showed easy-to-interpret conditional response probabilities than a 4-class solution, 
Table 1 Patient characteristics and multinomial logistic regression at assessment

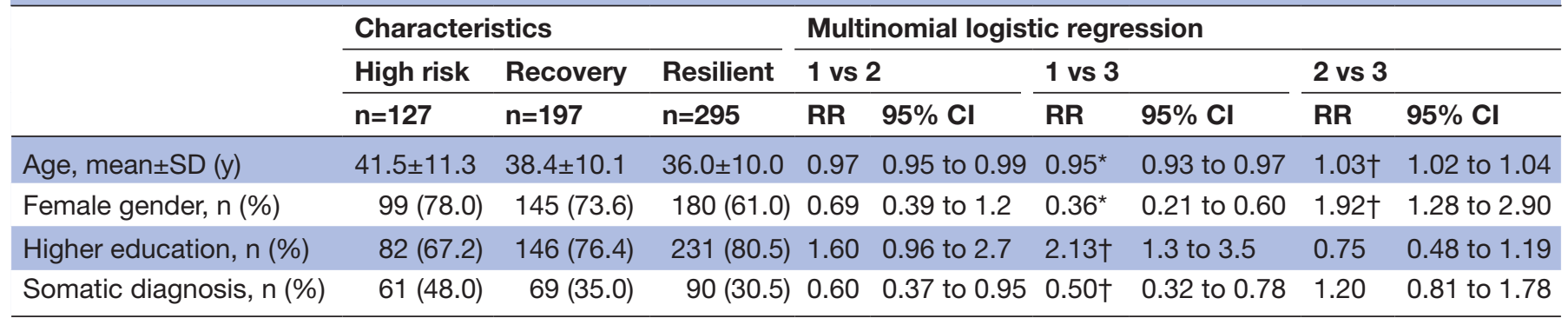

1: 'High risk', 2: 'Recovery', 3: 'Resilient'.

${ }^{*} \mathrm{P}<0.001$ level.

$\dagger P<0.01$ level.

$\mathrm{RR}$, Relative risk.

providing a more reasonable representation of the data and more parsimonious model.

For the first trajectory, sick leave at one year pretreatment was $10.8 \%$, at screening $67.4 \%$ and at one year post-treatment $47.5 \%$. Patients belonging to this trajectory class were labelled the 'High risk' group $(\mathrm{n}=127 ; 20.5 \%)$. For the second trajectory, sick leave at one year pre-treatment was $0.6 \%$, at screening it was $87.1 \%$, and at one year post-treatment it was $2.2 \%$. Patients belonging to this trajectory class were labelled the 'Recovery' group $(n=197 ; 31.8 \%)$. For the third trajectory, sick leave was low throughout the period, estimated sick leave was consistently below $4 \%$. Patients belonging to this trajectory were labelled the 'Resilient' group $(\mathrm{n}=295 ; 47.7 \%)$. At one year post treatment, $87.2 \% \quad(\mathrm{n}=540)$ of the total study population were fully working. Patients fully working one year post-treatment per trajectory were as follows: 'High risk' group, 48.8\% ( $\mathrm{n}=62)$; 'Recovery' group, $98.0 \%(\mathrm{n}=193)$; and for the 'Resilient' group $96.6 \%$ $(\mathrm{n}=285)$.

\section{Background and clinical characteristics of the groups}

Proportion of depressive disorder was largest in the 'Recovery' group (55.3\%) compared with the 'High risk' $(48.8 \%)$ and 'Resilient' groups 3 (42.4\%). There was no significant difference in waiting time or number of sessions between the groups, one-way
ANOVA $F(109,509)=1.16, \mathrm{p}=0.14$ and $F(19,599)=0.98$, $\mathrm{p}=0.47$, respectively. Half the patients $(49.9 \%)$ were on sick leave at the start of treatment, decreasing to $12.8 \%$ one year after the intervention. Multinomial logistic regression showed that there were no significant differences between the 'High risk' and 'Recovery' groups. The following covariates significantly predicted the log-odds of being in the 'High risk' group: higher age, female gender, not having higher education and having a previously diagnosed somatic illness. The results of the multinomial logistic regression are presented in table 1.

Figure 2 shows clinical scores pretreatment and post-treatment. Within-group (ES) from pretreatment to post-treatment were moderate to large on all measures. The lowest ES were consistently associated with the 'High risk' group $(d=0.54-0.84)$, while the 'Recovery' group had the highest ES on all measures $(d=1.10-1.81)$.

Table 3 shows a one-way ANOVA at pretreatment and post-treatment including Tukey's honestly significant difference (HSD) for between-group differences on clinical measures. Largest pretreatment differences were found on SRH and RTW-SE, $F(2,593)=38.17$, $\mathrm{p}=0.00$, and $F(2,596)=81.41, \mathrm{p}=0.00$, respectively. For between-group differences, the post hoc Tukey HSD showed that the 'High risk' and 'Recovery' groups were

Table 2 Model fit indices for latent growth mixture model $(\mathrm{N}=619)$

\begin{tabular}{|c|c|c|c|c|c|c|c|c|}
\hline & AIC & BIC & ABIC & Entropy & $\begin{array}{l}\text { LMR LR test } \\
p \text { value }\end{array}$ & $\begin{array}{l}\text { ALMR LR p } \\
\text { value }\end{array}$ & $\begin{array}{l}\text { Classification } \\
\text { accuracy }\end{array}$ & $\begin{array}{l}\text { BLRT p } \\
\text { value }\end{array}$ \\
\hline 1-Class & 6335.842 & 6353.561 & 6340.862 & - & & & - & - \\
\hline 3-Class & 5228.056 & 5290.072 & 5245.625 & 0.828 & 0.0424 & 0.0457 & 0.913 to 0.939 & $\star \star \star *$ \\
\hline 4-Class & 5124.340 & 5208.504 & 5148.182 & 0.828 & 0.0007 & 0.0008 & 0.853 to 0.948 & 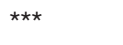 \\
\hline
\end{tabular}

***Denotes significant level at $p<0.001$.

ABIC, sample size adjusted BIC; AIC, Akaike information criterion; ALMR LR, Lo-Mendell-Rubin Adjusted Lrt Test; BIC, Bayesian information criterion; BLRT, bootstrap likelihood ratio test; LMR LR, Vuong-Lo-Mendell-Rubin Likelihood Ratio Test. 

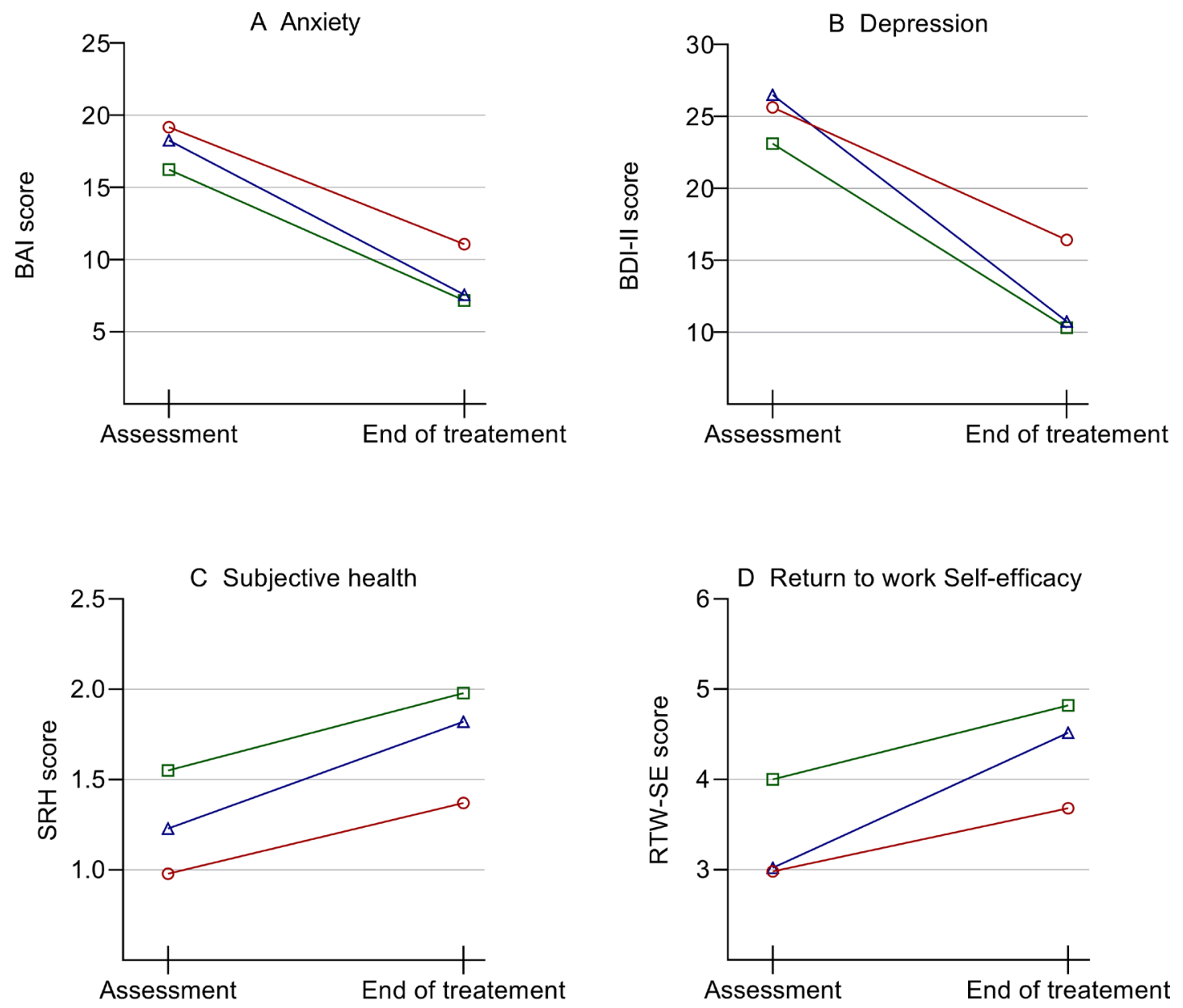

Figure 2 Effect size (ES) by Cohen's d. Anxiety: 'High risk': $d=0.74$, 'Recovery': $d=1.81$, 'Resilient' $d=1.04$. Depression: 'High risk': $d=0.84$, 'Recovery': $d=1.71$, 'Resilient' $d=1.41$. Subjective health: 'High risk': $d=0.54$, 'Recovery': $d=1.82$, 'Resilient' $\mathrm{d}=0.70$. RTW-SE: 'High risk': $d=0.66$, 'Recovery': $d=1.58$, 'Resilient' $d=0.98$. Cohen's $d>05$ indicates moderate ES, $>0.8$ indicates large ES. BAI, Beck Anxiety Inventory; BDI-II, Beck Depression Inventory-II; RTW-SE, return to work self-efficacy; $\mathrm{SRH}$, self-rated health.

only different on SRH at baseline. Post-treatment, the differences between the 'High risk' and 'Recovery' groups had increased markedly (table 3 , figure 2 ).

\section{DISCUSSION}

Our main aim was to determine if trajectories of sick leave could be detected in a population of patients with CMD who received work-focused treatment. We identified three groups with distinctly different sick leave trajectories. Almost half the patients ('Resilient', $47.7 \%$ ) had low sick leave throughout the 29.5 months, whereas the two remaining groups had either a near total RTW following treatment ('Recovery', 31.8\%), or sustained high sick leave ('High risk', 20.5\%). Our model showed good fit indices and was based solely on objective sick leave data from national registers spanning 2.5 years. We thus regard these groups to be robust classifications of sick leave behaviour in this patient cohort.

These subgroups and their characteristics have several implications. The 'Resilient' group largely avoided sick leave altogether despite relatively high symptom levels, on par with the two other groups. This suggests that there may be an element of prophylactic effect to the treatment. However, this is not a controlled study and we therefore do not know if their sick leave rates would have been higher without the intervention. We also do not know to which degree the 'Resilient' group experienced problems at work. Previous research would suggest at least some degree of presenteeism. ${ }^{2}$ Both are potential questions for future research.

At the start of treatment, $92.4 \%$ of the patients in the 'Recovery' group were on sick leave. This number steadily decreased for the remainder of the 
Table 3 Group differences on clinical measures at pretreatment and post-treatment $(\mathrm{N}=619)$

\begin{tabular}{|c|c|c|c|c|c|}
\hline & \multicolumn{2}{|c|}{ ANOVA } & \multicolumn{3}{|c|}{ Tukey HSD } \\
\hline & $F$ & $\eta^{2}$ & 1 vs 2 & 2 vs 3 & 1 vs 3 \\
\hline \multicolumn{6}{|l|}{ Assessment } \\
\hline BDI-II & $8.21^{*}$ & 0.025 & 0.79 & $3.86^{\star}$ & 2.49 \\
\hline $\mathrm{BAI}$ & 4.38 & 0.014 & 0.74 & 2.12 & 2.67 \\
\hline $\mathrm{SRH}$ & $38.17^{*}$ & 0.38 & $0.43 \dagger$ & $5.38^{\star}$ & $8.43^{*}$ \\
\hline RTW-SE & $81.41^{*}$ & 0.21 & 0.36 & $11.01^{*}$ & $9.90^{*}$ \\
\hline \multicolumn{6}{|c|}{ End of treatment } \\
\hline BDI-II & $19.96^{*}$ & 0.028 & $5.28^{*}$ & 0.49 & $6.07^{\star}$ \\
\hline BAI & $11.30^{*}$ & 0.011 & $3.87^{\star}$ & 0.54 & $4.61^{*}$ \\
\hline SRH & $43.00^{*}$ & 0.41 & $6.51^{*}$ & 2.62 & $9.25^{\star}$ \\
\hline RTW-SE & $73.02^{*}$ & 0.14 & $8.30^{\star}$ & $3.62^{*}$ & $12.07^{\star}$ \\
\hline
\end{tabular}

One-way ANOVA and Tukey honestly significant difference (HSD) test. 1: 'High risk', 2: 'Recovery', 3: 'Resilient'.

${ }^{*} \mathrm{P}<0.001$ level.

$\dagger \mathrm{P}<0.01$ level.

ANOVA, Analysis of variance; BAI, Beck Anxiety Inventory; BDIII, Beck Depression Inventory-II; RTW-SE, return to work selfefficacy; $\mathrm{SRH}$, self-rated health.

study's observational period. Within three months of completing treatment, $14.7 \%$ remained on sick leave, and at 12 months after the end of treatment, only $2 \%$ of the patients in this group remained on sick leave. A recent review defined sustainable RTW as no new sick leave within three months of initial RTW. ${ }^{36}$ The majority of patients who went on sick leave in this group can thus be said to have made a highly sustainable RTW.

Perhaps our most interesting findings are the contrasts and similarities between this 'Recovery' group and the 'High risk' group. These groups had similar initial sick leave trajectories, culminating in high sick leave at the start of treatment. During the treatment, however, their sick leave trajectories diverged sharply. The high rates of patients fully working in the 'Recovery' group was contrasted by the continued high sick leave of the 'High risk' group: $47.5 \%$ of patients in this group were still on sick leave 12 months after the end of treatment. In this respect, their sick leave prognosis at the start of treatment could hardly have been more different (Time 6 in figure 1). This was evident at the end of treatment but became even more pronounced three months after treatment had ended (Time 8 in figure 1). In fact, minimal changes in sick leave occurred after this time point. This implies that the probability of RTW is drastically reduced if it has not occurred within three months after ended treatment.

In the second aim of our study, we asked if the background or clinical characteristics at baseline could help distinguish between the groups. Differences in risk factors could help clinicians identify patients with poor sick leave prognosis and potentially tailor and adapt interventions accordingly. Significant differences were found on all background characteristics between the 'High risk' and 'Resilient' groups. Higher age, female gender, lower education and somatic comorbidity were all associated with higher risk of sick leave in our study. This is in line with previous research. ${ }^{13}$ However, no significant differences were found between the 'High risk' and 'Recovery' groups on background characteristics. Furthermore, clinical differences between the groups on anxiety and depression at baseline were small. Thus, it would likely not be possible for clinicians to predict sick leave prognosis at baseline in our study.

Despite similarities at baseline, clinical scores diverged for the 'High risk' and 'Recovery' groups during treatment, mirroring their sick leave trajectories. Although all three groups showed substantial clinical improvement, greatest change were seen in the 'Recovery' group and the smallest changes in the 'High risk' group. This was true of all clinical measures. Two scores are of particular interest. Firstly, the BDI-II showed that the depression score for the 'High risk' group remained above clinical threshold at the end of treatment, in line with previous research linking depression symptoms severity to delayed RTW. ${ }^{12}$ Secondly, the RTW-SE score of the 'High risk' group remained below the threshold that previous research has indicated is required for high probability of RTW. ${ }^{30}$ This implies that clinicians should be wary of residual depressive symptoms and low RTW-SE scores at the end of treatment.

A further implication is that these patients may need more follow-up. Clinicians could schedule future sessions or assess whether there are other services more appropriate for helping the patient recover. It is also possible that there are factors explaining the continued sick leave of the 'High risk' patients that were not recorded in the present study. Examples may include personal circumstance or characteristics of the work or workplace. As residual depressive symptoms predicted future sick leave, it is also worth noting that the wide-ranging impact of depression on a patient's life may not always be adequately recorded by standard clinical instruments. ${ }^{37}$ Future research investigating longitudinal sick leave trajectories in this patient group could thus benefit by a more thorough recording of workplace factors, and perhaps by supplementing quantitative measures with a qualitative approach to get a deeper understanding of the causes of continued sick leave.

Finally, it is worth mentioning that overall, the patient outcomes were generally favourable. In addition to clinical improvements in all groups, of the total sample, $87.2 \%$ of patients were fully working with no sick leave 12 months after the end of treatment. This is a marked increase compared with the start of treatment, where $50.1 \%$ of the total sample were fully working. Previous estimates indicate that a $5 \%$ increase in both the ability to work and productivity at work would mean a threefold return on investment in CMD treatment. ${ }^{38}$ The increase in work 
participation seen in the present study are well above these estimates.

\section{Strengths and limitations}

The longitudinal design of the study is a strength as sick leave and work status has been shown to fluctuate over time.$^{39}$ Other studies have used latent growth modeling to analyse longitudinal sick leave data after interventions. ${ }^{15} 16$ But to the best of our knowledge, ours' is the first study to also include data from before the intervention in classification of sick leave trajectories, thereby providing a much broader perspective on the issue. The sick leave data in question was objective data collected from registries with no loss to follow-up. In sum, this gave the study a robust classification of sick leave behaviour. The sample was also relatively large for a clinical study and was carried out in a national health service clinic, increasing ecological validity. Finally, a recent article using similar methodology emphasised the importance of developing better interventions grounded in a more thorough understanding of individual variation. Several factors that were highlighted as important areas of future research are included in the present study, including information on previous sick leave, comorbidity, psychological variables including self-efficacy, whether persons received an intervention or not and their specific diagnosis. ${ }^{40}$

Still, the observational design means that this study does not have a control group. For the future, a randomised controlled trial would be helpful to better understand the impact of the intervention on sick leave. As for the background and clinical data used in the post hoc analyses, no data were collected on workplace conditions. We do not know to which degree workplace issues affected outcomes. Future research on the topic would benefit from including workplace factors known to influence the RTW process, such as job sector, size of workplace and support from colleagues and supervisors. ${ }^{840}$ We also do not have clinical follow-up data for the year following treatment, and do not know if CMD relapse occurred, which could potentially explain the lower RTW rates in the 'High risk' group.'

Our findings should be interpreted with some caution. Overall, the variability in response to workfocused treatment has been demonstrated in numerous studies and is an obvious object of interest. ${ }^{11}$ However, the exploratory nature of our study and the inherent differences in welfare structures across countries indicates that one should be careful to generalise. Growth mixture modelling describes classes based on estimated posterior probabilities, which is then used to assign individuals to their most likely class membership. Growth mixture modelling is, therefore, exploratory, and as such more research is needed in replicating the classes that were identified in this study.

\section{Conclusion and future research}

The present study is part of a growing body of literature that uses latent growth modelling to analyse longitudinal sick leave data from patients suffering from anxiety and depression. The aim of these studies is to better understand the heterogeneity of these patients, with the hope of developing more tailored, and thus more effective, interventions. Our study found three groups with distinctly different sick leave trajectories. These trajectories were in turn associated with differences in background and clinical characteristics.

The two groups with high sick leave at the start of treatment had more women and higher age than the 'Resilient' group. However, gender and age did not differ between the two groups with high sick leave. Moreover, clinical symptoms of anxiety and depression at the start of treatment were similar across all three groups. Thus, neither background nor clinical characteristics could be used to predict sick leave prognosis at the start of treatment in this study. At the end of treatment, the 'High risk' group showed consistently poorer response than the two other groups on all clinical measures and also had residual depressive symptoms. A question for future research is thus to which degree long-term sick leave prognosis is linked to clinical outcomes at the end of treatment.

Should future research uncover similar groups with similar patterns, all groups identified in this study are of interest, for different reasons. The 'High risk' group can shed light on which factors maintain high sick leave despite treatment, and whether other interventions may be of more use. The 'Recovery' group may reveal factors that influence rapid RTW. The 'Resilient' group mostly avoided sick leave, despite symptoms levels comparable to the other groups. Future investigation of this group can help determine which factors act as protection against sick leave in the first place.

The findings in the present study point to the possibility of improving outcomes for patients on or at risk of sick leave due to anxiety and depression through stratifying and tailoring treatment.

Twitter Kenneth Sandin @kennethsandin

Acknowledgements We would like to thank patients, next of kin and user representatives for their participation and valuable feedback. We would also like to thank therapists and administrative staff at Diakonhjemmet Hospital for their participation and technical assistance.

Contributors KS led the writing of the manuscript and is the principle author of the funding application. FA contributed to data analyses and writing. KO was responsible for management and structuring of data. RGHG is the clinic leader and contributed to study design and intervention development. JSRL contributed to intervention development and writing. SER contributed to design and writing. $\mathrm{OH}$ is the project manager and contributed to design, analyses and writing. All authors contributed to, read and approved the final manuscript.

Funding The trial was mainly sponsored by Diakonhjemmet Hospital with additional funding from Southern and Eastern Norway Regional Health Authority, and Stiftelsen Dam (previously Extrastiftelsen), a not-for-profit trust that funds health research and innovation projects.

Competing interests None declared. 
Patient and public involvement Patients and/or the public were involved in the design, or conduct, or reporting, or dissemination plans of this research. Refer to the Methods section for further details.

Patient consent for publication Consent obtained directly from patient(s)

Ethics approval The study was approved by the Norwegian Data Protection Authority as health service research. The Norwegian Data Protection Authority has in such cases preapproved the use of data for research, and responsibility for secure and proper data management rests with the treatment provider, which in this case was Diakonhjemmet Hospital. There is therefore no file number or approval number or any similar reference for this type of research in Norway. This arrangement stipulates that written consent for the use of data for research must be obtained from each individual patient, which was done.

Provenance and peer review Not commissioned; externally peer reviewed.

Data availability statement Data are available upon reasonable request. Additional data can be accessed on request by email (kenneth.sandin@diakonsyk. no).

Open access This is an open access article distributed in accordance with the Creative Commons Attribution Non Commercial (CC BY-NC 4.0) license, which permits others to distribute, remix, adapt, build upon this work non-commercially, and license their derivative works on different terms, provided the original work is properly cited, appropriate credit is given, any changes made indicated, and the use is non-commercial. See: http://creativecommons.org/licenses/by-nc/4.0/.

ORCID iD

Kenneth Sandin http://orcid.org/0000-0002-1673-9606

\section{REFERENCES}

1 Steel Z, Marnane C, Iranpour C, et al. The global prevalence of common mental disorders: a systematic review and meta-analysis 1980-2013. Int J Epidemiol 2014;43:476-93.

2 OECD. Mental Health and Work. Switzerland - Mental health and work. Paris: OECD Publishing, 2014.

3 OECD. Health at a glance 2019: OECD indicators. Paris: OECD Publishing, 2019.

4 Vigo D, Thornicroft G, Atun R. Estimating the true global burden of mental illness. Lancet Psychiatry 2016;3:171-8.

5 OECD/EU health at a glance: Europe 2018: state of health in the EU cycle 2018.

6 Clark DM. Realizing the mass public benefit of evidence-based psychological therapies: the IAPT program. Annu Rev Clin Psychol 2018;14:159-83.

7 OECD. Mental health and work. Norway, 2013.

8 Cullen KL, Irvin E, Collie A, et al. Effectiveness of workplace interventions in return-to-work for musculoskeletal, pain-related and mental health conditions: an update of the evidence and messages for practitioners. J Occup Rehabil 2018;28:1-15.

9 Salomonsson S, Santoft F, Lindsäter E, et al. Cognitive-behavioural therapy and return-to-work intervention for patients on sick leave due to common mental disorders: a randomised controlled trial. Occup Environ Med 2017;74:905-12.

10 Reme SE, Grasdal AL, Løvvik C, et al. Work-focused cognitivebehavioural therapy and individual job support to increase work participation in common mental disorders: a randomised controlled multicentre trial. Occup Environ Med 2015;72:745-52.

11 Nigatu YT, Liu Y, Uppal M, et al. Interventions for enhancing return to work in individuals with a common mental illness: systematic review and meta-analysis of randomized controlled trials. Psychol Med 2016;46:3263-74.

12 Ervasti J, Joensuu M, Pentti J, et al. Prognostic factors for return to work after depression-related work disability: a systematic review and meta-analysis. J Psychiatr Res 2017;95:28-36.

13 de Vries H, Fishta A, Weikert B, et al. Determinants of sickness absence and return to work among employees with common mental disorders: a scoping review. J Occup Rehabil 2018;28:393-417.

14 Hultin H, Lindholm C, Malfert M, et al. Short-term sick leave and future risk of sickness absence and unemployment - the impact of health status. BMC Public Health 2012;12.

15 Hellström L, Madsen T, Nordentoft M, et al. Trajectories of return to work among people on sick leave with mood or anxiety disorders: secondary analysis from a randomized controlled trial. J Occup Rehabil 2018;28:666-77.
16 Arends I, Almansa J, Stansfeld SA, et al. One-year trajectories of mental health and work outcomes post return to work in patients with common mental disorders. J Affect Disord 2019;257:263-70.

17 Ubalde-Lopez M, Arends I, Almansa J, et al. Beyond return to work: the effect of multimorbidity on work functioning trajectories after sick leave due to common mental disorders. J Occup Rehabil 2017;27:210-7.

18 NICE. Depression in adults: recognition and management, 2009. Available: https://www.nice.org.uk/guidance/cg90

19 NICE. Generalised anxiety disorder in adults, in national clinical guideline number 113. London, 2019.

20 Dobson KS, Dozois DJA. Handbook of cognitive behavioral therapies. 4th edn. Guilford Press, 2019.

21 Wells A. Metacognitite therapy for anxiety and depression. New York, NY: Guildford Press, 2011.

22 Lagerveld SE, Blonk RWB, Brenninkmeijer V, et al. Workfocused treatment of common mental disorders and return to work: a comparative outcome study. J Occup Health Psychol 2012;17:220-34.

23 Gjengedal RGH, Reme SE, Osnes K, et al. Work-focused therapy for common mental disorders: a naturalistic study comparing an intervention group with a waitlist control group. Work 2021;66:657-67.

24 Norwegian Labour and Welfare Administration. Absence due to illness and sick-pay, 2018. Available: https://www.altinn.no/en/startand-run-business/working-conditions/print-sickness-and-leaves-ofabsence/absence-due-to-illness-and-sick-pay/ [Accessed 28 Jan 2020].

25 WHO. The International classification of diseases-10 (ICD-10) Geneva: World Health Organisation, 1992.

26 Nigatu YT, Liu Y, Uppal M, et al. Prognostic factors for return to work of employees with common mental disorders: a meta-analysis of cohort studies. Soc Psychiatry Psychiatr Epidemiol 2017;52:1205-15.

27 Beck AT, Steer RA, Brown GK. Manual for beck depression inventory II (BDI-II). San Antonio: Psychology Corporation, 1996.

28 Beck AT, Epstein N, Brown G, et al. An inventory for measuring clinical anxiety: psychometric properties. J Consult Clin Psychol 1988:56:893-7.

29 Schnittker J, Bacak V. The increasing predictive validity of self-rated health. PLoS One 2014:9:e84933.

30 Gjengedal RGH, Lagerveld SE, Reme SE, et al. The return-to-work self-efficacy questionnaire (RTW-SE): a validation study of predictive abilities and cut-off values for patients on sick leave due to anxiety or depression. J Occup Rehabil 2021;31:664-73.

31 Muthén LK, Muthén BO. Mplus user's guide. Seventh ed. Los Angeles, CA: Muthén \& Muthén, 1998-2012. https://www.statmodel. com/download/usersguide/Mplus\%20user\%20guide\%20Ver_7_r6_ web.pdf

32 Heck RH, Thomas SL. An introduction to multilevel modeling techniques: MLM and SEM approaches using MPLUS. Routledge, 2015.

33 Muthén B. Statistical and substantive checking in growth mixture modeling: comment on Bauer and Curran (2003). Psychol Methods 2003:8:369-77.

34 Clark SL, Muthén B. Relating latent class analysis results to variables not included in the analysis, 2012. Available: https:// www.statmodel.com/download/relatinglca.pdf [Accessed 8 Jan 2020].

35 Cohen J. Statistical power analyses for the social sciences. Hillsdale, NJ: Lawrence Erlbauni Associates, 1988.

36 Etuknwa A, Daniels K, Eib C. Sustainable return to work: a systematic review focusing on personal and social factors. J Occup Rehabil 2019;29:679-700.

37 Chevance A, Ravaud P, Tomlinson A, et al. Identifying outcomes for depression that matter to patients, informal caregivers, and healthcare professionals: qualitative content analysis of a large international online survey. Lancet Psychiatry 2020;7:692-702.

38 Chisholm D, Sweeny K, Sheehan P, et al. Scaling-up treatment of depression and anxiety: a global return on investment analysis. Lancet Psychiatry 2016;3:415-24.

39 Øyeflaten I, Lie SA, Ihlebæk CM, et al. Multiple transitions in sick leave, disability benefits, and return to work. - A 4-year follow-up of patients participating in a work-related rehabilitation program. BMC Public Health 2012;12:748.

40 Spronken M, Brouwers EPM, Vermunt JK, et al. Identifying return to work trajectories among employees on sick leave due to mental health problems using latent class transition analysis. BMJ Open 2020;10:e032016. 